\title{
Catalysis inside the Hexameric Resorcinarene Capsule
}

Qi Zhang ${ }^{a}$, Lorenzo Catti ${ }^{a}$, Konrad Tiefenbacher*a,b

a. Department of Chemistry, University of Basel, BPR 1096, PO Box 3350, Mattenstrasse 24a, CH-4002 Basel, Switzerland

b. Department of Biosystems Science and Engineering, ETH Zurich, Mattenstrasse 26, CH-4058

Basel, Switzerland

Conspectus: In this Account, we outline our investigation into the supramolecular resorcinarene capsule as a catalyst. Molecular capsules are not only of interest due to the similarities of their binding pockets with those of natural enzymes but also feature potential advantages for catalysis. Due to the restricted internal volume of the binding pockets, substrate selectivities are commonly observed. Substrates that are encapsulated more efficiently will be converted selectively in the presence of less suitable substrates. This size selectivity cannot be obtained in a regular solution experiment. In addition, because of the distinct chemical environment inside the capsule, different product selectivities may be observed. Furthermore, the encapsulation of reactive catalysts inside confined environments may improve catalyst compatibility for multicatalyst tandem reactions. 
Although the potential advantages of performing catalysis inside closed microenvironments are generally recognized, the number of known catalytically active supramolecular host systems is still very limited. There are several reasons, the most important of which is that it is very difficult to predict the catalytic potential of known supramolecular host systems. In several cases, even the encapsulation behavior of host systems is not completely understood or explored. Therefore, it is evident that further research is required to explore the potential of catalysis inside supramolecular capsules.

Our initial research mainly focused on understanding the puzzling encapsulation behavior of the self-assembled resorcinarene capsule I and the closely related pyrogallolarene capsule II. After the elucidation of the decisive differences between these two systems, we explored the catalytic potential of capsule I. A variety of different reactions was successfully performed inside its cavity. The most important examples highlighted in this article are iminium catalysis, the tail-to-head terpene cyclization and the carbonyl-olefin metathesis. In the case of proline-mediated iminium catalysis, we were able to demonstrate that the enantioselectivity for the product formation was increased when the reaction was performed inside the cavity of capsule $\mathbf{I}$. This is remarkable since the capsule is formed from achiral building blocks and, therefore, not adding chiral information to the reaction mixture. The tail-to-head terpene cyclization is the most complex reaction performed so far inside capsule $\mathbf{I}$. The cyclic monoterpenes eucalyptol and $\alpha$-terpinene were formed in useful yields. Interestingly, these products have not yet been synthetically accessible in solution directly from acyclic terpene precursors. Furthermore, we demonstrated that the cocatalytic system of capsule I and $\mathrm{HCl}$ is suitable for carbonyl-olefin metathesis. $\mathrm{HCl}$ was shown to be an inefficient catalyst for this reaction in solution experiments. This demonstrates that the different chemical environment inside the supramolecular container can lead to altered product selectivity. In general, 
we hope to demonstrate in this article that research of catalysis inside supramolecular capsules, although still in its infancy, is starting to produce first synthetically relevant results.

\section{Introduction}

Enzymes, nature's catalysts, have been serving as an inspiration for chemists due to their catalytic efficiency. ${ }^{1-2}$ Especially, their ability to accelerate reactions by many orders of magnitude under mild conditions and to produce products with excellent regio- and stereocontrol is fascinating. Since Linus Pauling's idea that transition state stabilization ${ }^{3}$ is a hallmark feature of enzymes, much effort has been devoted to understanding how enzymes actually work. The discussion is still ongoing but electrostatic interactions likely play a key role. ${ }^{4-5}$ Chemists initially tried to mimic the basic working principle of enzymes with preorganized open or macrocyclic structures. ${ }^{1-2}$ In the last decades, supramolecular chemists have shifted the attention towards more closed structures, so called molecular capsules. ${ }^{6-19}$ Such containers feature a defined cavity where substrates can bind in analogy to the binding sites of enzymes. One of the few supramolecular capsules which has been successfully exploited for the catalysis of a wide range of reactions is the resorcinarene hexamer I, originally reported by the Atwood research group. ${ }^{20}$ It self-assembles via hydrogen bonds from six resorcinarene units $\mathbf{1}$ and eight water molecules in apolar solvents like chloroform and benzene..$^{21-22}$ It does not feature large openings at the surface. Therefore, substrate uptake is believed to occur via the dissociation of one resorcinarene unit. ${ }^{23}$ Ammonium salts are well encapsulated inside the cavity of capsule $\mathbf{I}$, most likely due to cation- $\pi$ interactions with the aromatic walls of the container. ${ }^{21,24}$ Interestingly, it was also reported that it binds tertiary amines, although there should not be strong interactions with the cavity. ${ }^{25-26}$ 
Scheme 1. Structures of resorcinarene $\mathbf{1}$ and pyrogallolarene $\mathbf{2}$, and their respective self-assembled molecular capsules I and II. In capsule I, four out of the eight water molecules in the hydrogen bond network feature a free hydrogen bond donor site (three highlighted with purple circles, the fourth one is hidden by the central water molecule in the front). In the structures of the capsules, the undecyl groups are replaced by methyl groups for clarity.
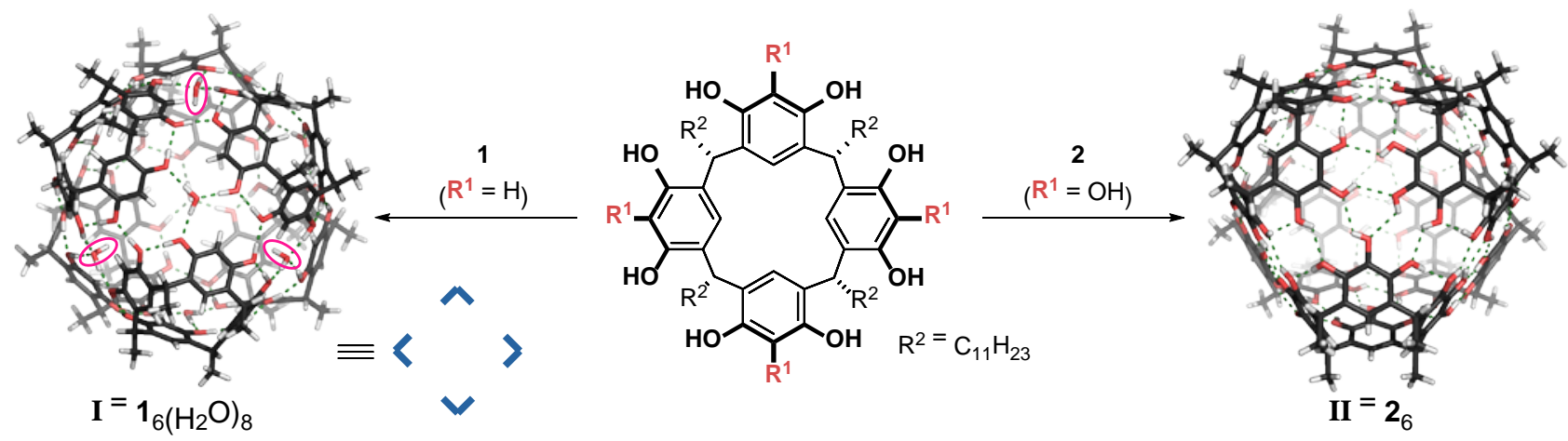

The very closely related molecular capsule II, originally reported by Mattay ${ }^{27}$ self-assembles from six pyrogallolarene units $\mathbf{2}$. In contrast to capsule $\mathbf{I}$, it does not require water to complete its hydrogen bond network. Surprisingly, capsule II does not bind ammonium salts but does bind tertiary amines. ${ }^{25-26}$ However, encapsulated amines were expelled upon the addition of acid. These seemingly contradicting observations were also identified as a "mystery" in a review article about the hexameric capsules in $2011 .^{22}$ As it turned out, research in our laboratory solved this puzzle and, subsequently, led to the discovery of the catalytic potential of capsule I for a series of reactions. However, let us start chronologically.

\section{Our Investigations}


Motivation. Our research group became interested in capsule I for several reasons: (1) The groups of Scarso and Reek reported in 2011 that capsule $\mathbf{I}$ is able to encapsulate a gold(I) catalyst. ${ }^{28}$ They demonstrated that the entrapped catalyst displays different product selectivities when operating inside this closed environment, although at a reduced reaction rate. (2) The puzzling observation that capsule I binds tertiary amines as strong as ammonium salts. Moreover, that capsule II binds amines but not ammonium salts. (3) The ready availability of capsule $\mathbf{I}$ since resorcinarene $\mathbf{1}$ is synthetically accessible in one single step without the need for chromatography. (4) The unusually large inner volume of capsule I (approx. $1400 \mathrm{~A}^{3}$ ). It is large enough for the encapsulation of a wide range of substrates. For instance, ammonium salts as large as tetraoctylammonium bromide can be encapulated. ${ }^{23,24}$ (5) We speculated that iminium chemistry should be feasible inside capsule I due to its strong affinity for ammonium salts.

\section{Understanding the differences between capsule I and II}

Our investigations started with the study of amine uptake inside capsule I. Titration experiments with triethylamine, studied by ${ }^{1} \mathrm{H}$ NMR spectroscopy, revealed that a proton transfer from capsule I onto the amine is responsible for its uptake. ${ }^{29}$ The formed ammonium species is stabilized inside capsule I due to ion-ion and cation- $\pi$ interactions. The negative charge on the capsule surface is delocalized over the hydrogen bond network as indicated by ${ }^{1} \mathrm{H}$ NMR spectra and also DFT calculations. The acidity of the hexamer I was estimated by titration experiments with bases of varying basicity. A surprisingly high acidity ( $\mathrm{p} K_{\mathrm{a}}$ of 5.5-6) was determined for capsule $\mathbf{I}$. Recent DFT calculations by the groups of Rescifina and Gaeta confirmed this mean pKa value and found that there are four localized zones with a microenvironmental $\mathrm{p} K_{\mathrm{a}}$ of approx. $2.5 .^{30}$ These zones correspond to the four water molecules integrated into the hydrogen bond network of $\mathbf{I}$ that feature a free hydrogen bond donor (see purple markings in Scheme 1). 
Subsequently, we identified that protonation of tertiary amines also occurs inside capsule II, although its acidity is much lower ( $\mathrm{p} K_{\mathrm{a}}$ of $\left.9.5-10\right)$. This explained the uptake of amines and indicated that cations are stabilized inside this system. ${ }^{31}$ But then why do ammonium salts resist encapsulation inside $\mathbf{I I}$ ? It was found that beside cation- $\pi$ stabilization, capsule $\mathbf{I}$ is also able to bind the counterion of ammonium cations. Evidence for counterion encapsulation was obtained from the NMR experiments using mesylate as the counterion. The four water molecules that function as single $\mathrm{H}$-bond donors in the H-bond network are able to stabilize anions via H-bonds. This stabilization of anions is lacking in capsule II, which does not feature water in its H-bond network. This finally explained the surprising encapsulation behavior of capsule II: tertiary amines are bound in their protonated state and the counterion is the negatively charged capsule. Upon addition of external acid, for instance hydrochloric acid ( $\mathrm{HCl})$, the negatively charged capsule is protonated and an ion pair of protonated amine and chloride anion is formed. This ion pair is not a good guest for capsule II anymore since the anion cannot be properly stabilized, in contrast to capsule $\mathbf{I}$. Therefore, the ion pair is released.

Our investigations into the encapsulation behavior of capsule I and II not only clarified the puzzling encapsulation behavior, but also encouraged investigations into catalysis. Due to the discovered moderate Brønsted acidity of capsule $\mathbf{I}$ and its ability to stabilize cationic species via cation- $\pi$ interactions, we became interested in the exploration of reactions with cationic transition states.

\section{Catalytic applications}

Acetal hydrolysis. After having identified the acidity of hexamer I that is responsible for the good uptake of tertiary amines, we tried to translate this finding to catalytic applications. As a simple 
test reaction, we chose acetal hydrolysis. ${ }^{29}$ In the presence of catalytic amounts of capsule $\mathbf{I}$ (10 mol\%), hydrolysis of small diethyl acetals like 3 (Scheme 2) was rapid, while larger derivatives like acetal 4 were hydrolyzed much slower. This was consistent with a reaction on the inside of the capsule where smaller substrates are encapsulated more efficiently than larger ones. In addition, after blocking the cavity with the high-affinity guest tetrabutylammonium bromide, the reaction of the small substrate $\mathbf{3}$ was efficiently suppressed. Admittedly, acetal hydrolysis is not an exciting reaction and can be readily performed in solution. However, if the reaction is indeed catalyzed only inside the container under these conditions, a size selective reaction should be feasible. Indeed, the smaller acetal $\mathbf{3}$ is hydrolyzed with excellent selectivity in the presence of the larger acetal 4, to produce mainly acetaldehyde 5 (98:2 selectivity, Scheme 2). As expected, in solution using trifluoroacetic acid as the catalyst, no significant selectivity was observed. This result highlights one of the advantages ${ }^{9}$ of performing chemistry inside supramolecular capsules: size selectivity. Later investigations in our group uncovered that trace amounts of $\mathrm{HCl}$ are required for the hydrolysis inside the capsule. ${ }^{32}$ Although precautions were taken to exclude traces of $\mathrm{HCl}$ from the solvent chloroform (filtration through basic aluminium oxide), it turned out that resorcinarene 1, prepared under aqueous acidic conditions, contains traces of $\mathrm{HCl} .{ }^{33}$ Nevertheless, catalysis takes place inside the container and $\mathrm{HCl}$ functions only as a cocatalyst. $\mathrm{HCl}$ alone under such conditions $(0.1 \mathrm{~mol} \% \mathrm{HCl})$ is not able to hydrolyze the acetals. ${ }^{32}$

Scheme 2. Size selective acetal hydrolysis catalyzed inside capsule I. 


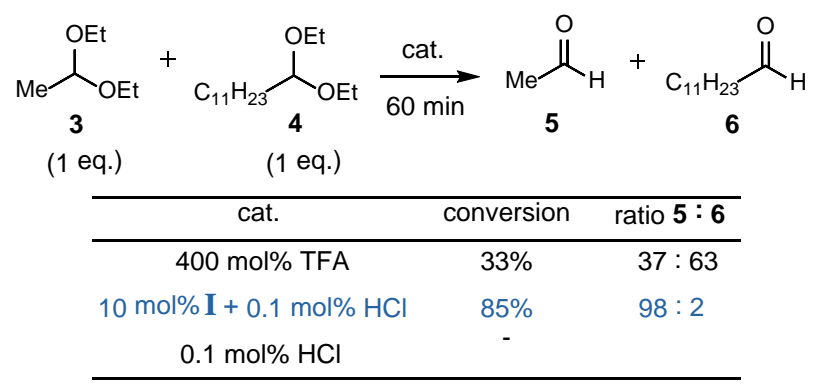

Iminium catalysis. As mentioned earlier, our interest in capsule $\mathbf{I}$ was also sparked by the idea to influence iminium-catalyzed reactions via encapsulation of the iminium ion. Due to the high affinity of capsule $\mathbf{I}$ for ammonium salts, we speculated that also iminium species should be encapsulated well. In asymmetric iminium catalysis, an $\alpha, ß$-unsaturated aldehyde (7, Scheme 3$)$ is condensed with a chiral optically active secondary amine catalyst $\mathbf{8}$, to produce the activated iminium electrophile $\mathbf{9} \cdot{ }^{34}$ If the formed iminium species $\mathbf{9}$ is encapsulated quantitatively to produce complex $\mathbf{A}$, the addition of the nucleophile would have to take place inside the confined space of capsule I. Therefore, different selectivities might be observable inside the capsule than in a regular solution experiment. After hydrolysis of the formed enamine (complex $\mathbf{C}$ ) via complex $\mathbf{D}$, the product should be released to close the catalytic cycle. Several reactions were investigated and the 1,4-reduction using Hantzsch ester $\mathbf{1 1}$ as a formal hydride source was chosen as a model reaction (Figure 1a). We were able to demonstrate that indeed intriguing differences in enantioselectivity were occurring in the presence and absence of catalytic amounts of capsule $\mathbf{I}^{35-36}$ For instance, the use of L-proline (20 mol\%), a poorly performing catalyst for iminium chemistry, delivers unsurprisingly only $9 \pm 2 \%$ ee $(S)$ in the solution experiment. However, if capsule $\mathbf{I}$ is present, the product is obtained with much higher enantioselectivity, $74 \pm 0 \%$ ee (S). Several control experiments indicated that the modulation of enantiomeric excess indeed stems from an 
encapsulation effect. In other words, this strong modulation effect is only observed if the reaction takes place inside capsule $\mathbf{I}$. This is especially remarkable when considering that capsule $\mathbf{I}$ just forms from achiral building blocks $\mathbf{1}$ and water molecules. Although the assembly $\mathbf{I}$ is chiral due to the twisted orientation of the resorcinarene units, ${ }^{20}$ it is of course only present in racemic form. What could then cause this modulation of enantioselectivity ( $\Delta e e$ of $65 \%$ ) inside capsule I? One potential explanation may lie in the preferential binding of the iminium species from the less hindered side - anti to the carboxylic acid - to the inner capsule walls (see Fig. 1b). This would mainly leave the top face for attack by the nucleophile and would deliver, as observed, the $S$ enantiomer preferentially. Alternatively, the chiral amine/iminium species may also impose optical activity onto capsule $\mathbf{I},{ }^{37}$ although this explanation seems less likely to us since we would not expect such a large effect in this case. Interestingly, capsule II did not display any significant modulation of enantioselectivity for this reaction (Fig. 1a). At first, this might seem surprising. But the iminium species $\mathbf{9}$ is present as an ion pair in the relatively apolar solvent chloroform (the chloride counterion stems from the $\mathrm{HCl}$ formed via photodegradation of chloroform), and as discussed before, capsule II does not bind ion pairs well due to its inability to stabilize anions. Therefore, the failure of capsule II to encapsulate ion pairs is the most likely explanation for its inability to influence iminium catalysis.

Further investigation into the iminium-catalyzed reaction inside I revealed that ortho-substituted derivatives of 7a display even more pronounced modulation effects (Figure 1c). For instance in the case of the $o$-methyl derivative $7 \mathbf{b}$, a $\Delta e e$ of $92 \%$ was observed. Additionally, benzothiazolidines of the general structure 12 (Figure 1d) were investigated as alternative hydride donors. ${ }^{36}$ The results obtained indicate that the substituent ' $R$ ' on the benzothiazolidine plays a crucial role for the selectivity observed inside the capsule. In the case of phenyl-substitution a 
reversal in selectivity was observed and the $(R)$-product formed preferentially inside the capsule in the presence of L-proline as chiral catalyst. The exact origin of this reversal is not clear yet but non-covalent interactions with the phenyl ring that lead to different binding modes seem most likely.

Scheme 3. General overview of iminium catalysis inside capsule I. Catalytic amounts of capsule I should be sufficient to encapsulate the iminium ions produced in the catalytic cycle.

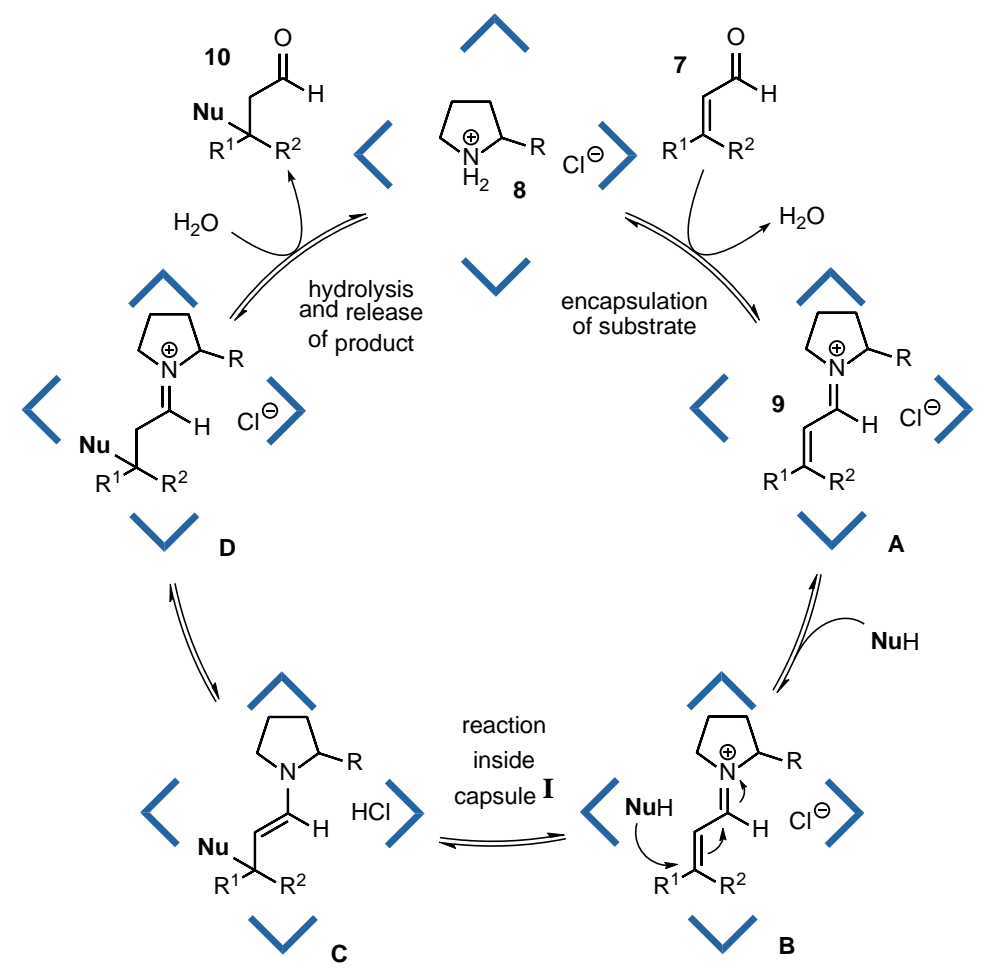


a)<smiles>C/C(=C\C=O)c1ccccc1</smiles>

$7 a$

1 equiv<smiles>CCOC(=O)C1=C(C)NC(C)=C(C(=O)OCC)C1</smiles>

11

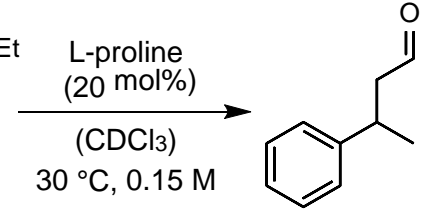

$10 a$
1.5 equiv

\begin{tabular}{|c|c|c|c|c|c|}
\hline Entry & Capsule & $\begin{array}{l}\text { Yield } \\
(\%)\end{array}$ & $\begin{array}{l}\text { enantiomeric } \\
\text { excess }\end{array}$ & $\Delta e e$ & $\Delta \Delta G^{\neq}$ \\
\hline
\end{tabular}

\begin{tabular}{|c|c|c|c|c|c|}
\hline 1 & $\mathbf{I}_{(26}$ mol\%) & $93 \pm 1$ & $74 \pm 0 \%$ ee $(S)$ & \multirow[b]{2}{*}{$65 \%$} & $4.79 \mathrm{KJ} / \mathrm{mol}$ \\
\hline & & & & & \\
\hline 2 & --- & $27 \pm 0$ & $9 \pm 2 \%$ ee $(S)$ & \multirow[b]{2}{*}{$\sim 0 \%$} & $0.45 \mathrm{KJ} / \mathrm{mol}$ \\
\hline 3 & II $_{(26 \text { mol\%) }}$ & $78 \pm 3$ & $9 \pm 1 \%$ ee $(S)$ & & $0.45 \mathrm{KJ} / \mathrm{mol}$ \\
\hline
\end{tabular}

b)

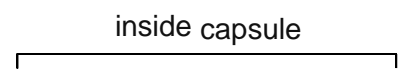

(S)-product

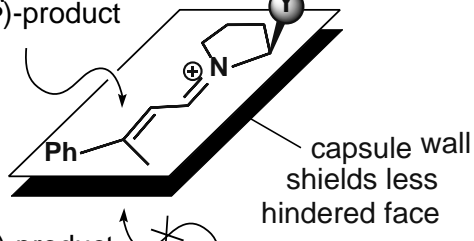

$(R)$-product $\nsucc$ hindered face

d)<smiles>[R]C1Nc2ccccc2S1</smiles>

12

c)

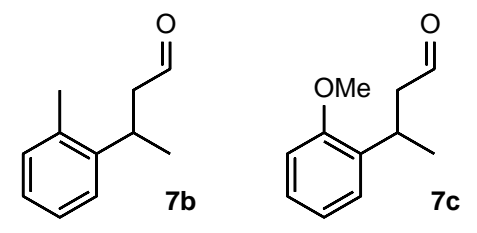<smiles>CC(CC=O)c1ccccc1F</smiles><smiles>CC(CC=O)c1ccccc1Cl</smiles><smiles>CC(CC=O)c1ccccc1Br</smiles>

$\Delta \Delta G^{\neq}$(with $\mathbf{I}$ ) $\quad 4.68 \mathrm{KJ} / \mathrm{mol}$

$5.27 \mathrm{KJ} / \mathrm{mol}$

$5.27 \mathrm{KJ} / \mathrm{mol}$

$5.14 \mathrm{KJ} / \mathrm{mol}$

$4.27 \mathrm{KJ} / \mathrm{mol}$

$\Delta \Delta G^{\neq}$(without $\mathbf{I}^{\text {) }} \quad-0.97 \mathrm{KJ} / \mathrm{mol}$

$0.45 \mathrm{KJ} / \mathrm{mol}$

$0.20 \mathrm{KJ} / \mathrm{mol}$

$-0.15 \mathrm{KJ} / \mathrm{mol}$

$0.15 \mathrm{KJ} / \mathrm{mol}$

$\Delta$ ee

$92 \%$

$69 \%$

$74 \%$

$80 \%$

$66 \%$

Figure 1. Iminium catalysis inside capsule I. a) The 1,4-reduction of aldehyde 7a was performed in the presence and absence of capsule I and II. $\Delta$ ee is defined as the difference between the enantiomeric excess obtained from the reaction in the presence and in the absence of capsule $\mathbf{I}$. $\Delta \Delta \mathrm{G}^{*}$ is defined as the difference between the energy barriers for the formation of the $R$ - and the $S$-product, respectively $\left(\Delta \Delta \mathrm{G}^{\neq}=\Delta \mathrm{G}_{R}^{\neq}-\Delta \mathrm{G}_{S}^{\neq}\right)$. b) Binding of the iminium ion to the inner capsule walls from the less hindered side may explain the increased enantioselectivities observed inside capsule I. c) Ortho-substituted substrates displayed an increased difference in enantioselectivity. d) The benzothiazolidines of the general structure $\mathbf{1 2}$ were investigated as alternative hydride donors. 
Terpene cyclizations. In our eyes, the most fascinating example for catalysis inside capsule $\mathbf{I}$ is the tail-to-head ${ }^{38}$ terpene cyclization (Scheme 4). This reaction enables nature to build up the large and structurally diverse group of terpene natural products from just a few simple acyclic terpenes. In contrast to the head-to-tail terpene cyclization that has been successfully reproduced in solution, ${ }^{39}$ man-made catalysts for the more challenging tail-to-head cyclization are lacking. One main issue is that regular Lewis or Brønsted catalysts lack the ability to influence the conformation of the flexible acyclic terpene precursor (e.g. nerol 13, Scheme 4a) in a meaningful way. Therefore, it is necessary to develop catalysts with binding pockets that potentially allow control over the substrate conformation. Inspiration to investigate this reaction class came from reports that aromatic residues play a key role in stabilizing cationic intermediates and transition states in natural cyclase enzymes via cation- $\pi$ interactions. ${ }^{40-41}$ Since cationic species are bound well inside I, investigation of this reaction class seemed obvious, although we expected limited prospects of success with this simple system. To our surprise, initial experiments of commercially available nerol already led to a tail-to-head cyclization with eucalyptol (16) as the main product (39\%, see Scheme $4 \mathrm{~b}){ }^{42} \mathrm{~A}$ series of alternative leaving groups was investigated and acetate turned out to be well suitable. For instance, the cyclization of geranylacetate (GOAc, 17) inside I yielded mainly $\alpha$-terpinene (19,35\%). The cyclization to $\alpha$-terpinene seems to be a "non-stop" cyclization as we were not able to detect intermediates. In contrast to natural enzymes which bind intermediates strongly, capsule I does not retain neutral intermediate products and allows their detection by NMR and gas chromatography. A detailed investigation revealed that more polar/functionalized leaving groups that bind stronger to the cavity via H-bonds and/or $\pi-\pi$ interactions display an altered selectivity. ${ }^{43}$ Further studies revealed that the observed catalytic activity depends on the synergistic interplay between capsule I and $\mathrm{HCl}$. No cyclization reaction was observed when either capsule I 
or $\mathrm{HCl}$ was omitted. Much higher concentrations of $\mathrm{HCl}$ were required to observe a reaction in solution and led to the formation of a different cyclization product ( $\alpha$-terpinyl chloride). A series of control experiments indicate that that the reaction occurs inside the cavity. When capsule I was blocked by a strongly binding inhibitor ( $n \mathrm{Bu} 4 \mathrm{NBr})$, only trace amounts of cyclization products were formed. One of the strongest control experiments is the competition experiment between geranylacetate and its elongated derivative 20 of comparable reactivity (Scheme 4c). The larger substrate which is not encapsulated as efficiently as GOAc, is converted much slower (after $24 \mathrm{~h}$ only $2 \%$ conversion as compared to $81 \%$ conversion of GOAc). This pronounced size selectivity provides very strong evidence that the reaction is indeed accelerated inside the capsule.

The cyclization of GOAc was investigated in detail, in order to learn more about the catalytic cycle and the rate-limiting step. ${ }^{1} \mathrm{H}$ NMR experiments indicate a fast protonation of the capsule when the cocatalyst $\mathrm{HCl}$ is present in solution. Evidence for substrate uptake was also obtained by ${ }^{1} \mathrm{H}$ NMR experiments. Our current hypothesis is that the substrate is then activated via protonation. The cleavage of the leaving group was found to be the rate-limiting step. The measured positive entropy of activation, as well as the normal secondary isotope effect ruled out other possibilities. After isomerization of the transoid allylic cation to its cisoid conformation (18), cyclization can take place. This is most likely followed by a 1,2 or 1,3 hydride shift and subsequent proton elimination to produce $\alpha$-terpinene. Due to the less polar nature of the cyclization product as compared to the starting material, product release is facile. Therefore, product inhibition was not observed. 
Scheme 4. Tail-to-head terpene cyclizations performed inside capsule I. a) The cyclization of nerol (13) produces eucalyptol (16) as the major product. b) Geranyl acetate (17) undergoes selective „non-stop“ cyclization to yield $\alpha$-terpinene (19). c) The selective converison of geranyl acetate (17) in the presence of its extended analogue $\mathbf{2 0}$ provides strong evidence that the cyclization occurs inside capsule $\mathbf{I}$.

a)

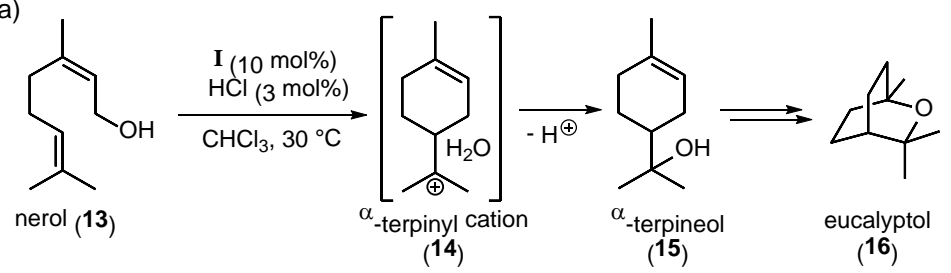

b)

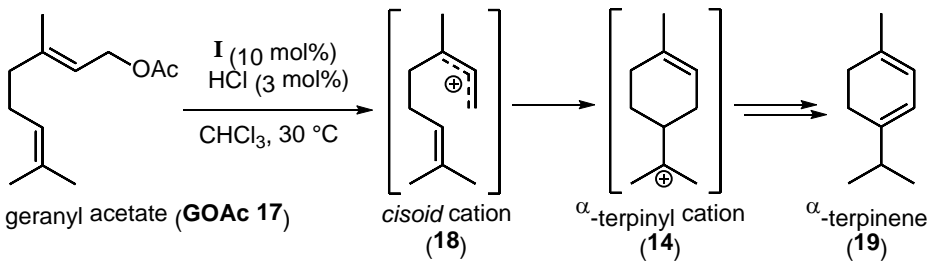

c)

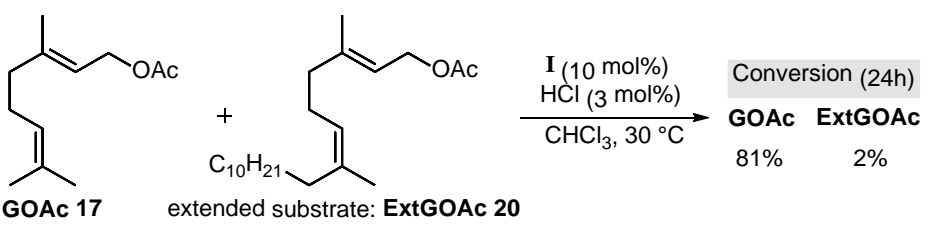

Currently, we are investigating the cyclization of more complex sesquiterpenes, containing fifteen carbon atoms, inside the cavity of $\mathbf{I}$. These studies will be reported in due course.

We also looked at related reactions. The formation of cyclic ethers like eucalyptol is more general and different six- and seven-membered rings can be formed in good to excellent yields inside capsule I ${ }^{44}$ Related terpene-like cyclization of tertiary alcohols/acetates were also investigated and yielded complex cyclopentene structures and in one case even a cyclobutanone. ${ }^{45}$ For more details, we refer the reader to the respective publications. 
Carbonyl-olefin metathesis. Very recently, we applied the cocatalytic system of capsule I and $\mathrm{HCl}$ to the carbonyl-olefin metathesis (Scheme 5). ${ }^{46}$ In 2016, the Schindler group demonstrated that $\mathrm{FeCl}_{3}$ is a competent catalyst for this type of reaction. ${ }^{47}$ Our interest was mainly sparked because it was reported that Brønsted acids like $\mathrm{HCl}$ are not suitable for this reaction in solution. ${ }^{48}$ We wondered if that limitation can be overcome by using capsule $\mathbf{I}$ as a reaction vessel. Indeed, the reaction was successfully realized using $10 \mathrm{~mol} \%$ of $\mathbf{I}$ and $5 \mathrm{~mol} \%$ of $\mathrm{HCl}$. The control experiments performed indicate that indeed both catalyst components are required and that the reaction takes place inside the cavity of I. For instance, blocking the cavity with the strongly binding tetrabutylammonium bromide inhibited the reaction. Furthermore, differently sized substrates with similar reactivity in solution displayed contrasting reactivity in the presence of the capsule. The smaller substrate that is encapsulated more efficiently is converted much faster than the larger counterpart. The substrate scope was explored and it was found that especially $\delta, \varepsilon-$ unsaturated ketones like 21a-c were converted in much higher yields to the corresponding cyclopentenes as compared to the solution benchmark catalyst $\mathrm{FeCl}_{3}$. Preliminary mechanistic investigations indicated that the oxetane intermediate is likely formed in a step-wise fashion. Detailed mechanistic investigations, as well as exploration of intermolecular carbonyl-olefin metathesis reactions are currently under way.

Scheme 5. The carbonyl-olefin metathesis was explored inside capsule I. Especially ketone substrates like 21a-c were converted more efficiently with the $\mathrm{HCl} / \mathrm{capsule}$ I system as compared to the benchmark solution catalyst $\mathrm{FeCl}_{3}$. 

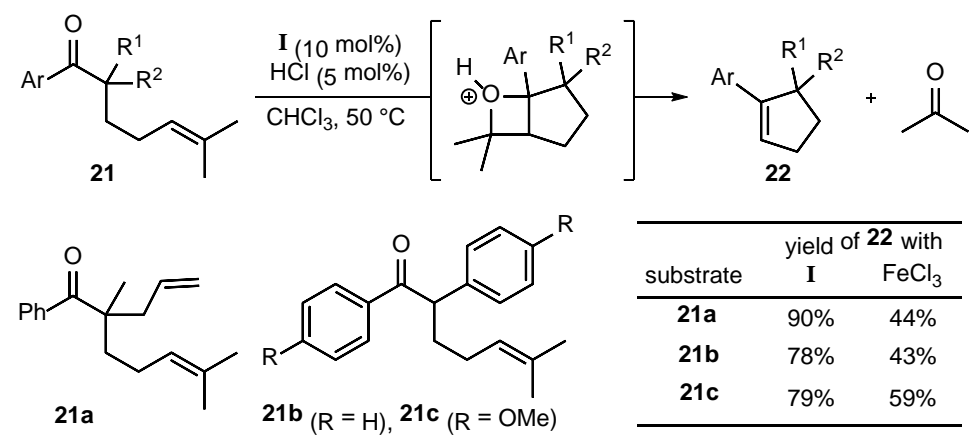

Prerequisites for catalytic activity. The ability to stabilize cationic intermediates and transition states has been postulated as a main reason for the observed acceleration of reactions inside capsule I and related supramolecular containers. Surprisingly, capsule II proved inactive in catalyzing reactions with cationic transition states, although substrates were encapsulated. Initially, due to the different acidities of capsule I ( $\mathrm{p} K_{\mathrm{a}}$ of 5.5-6) and II (pKa of 9.5-10), the inability of capsule II to protonate the substrate seemed to be the likely cause for its catalytic inability. ${ }^{31}$ However, since a strong external acid $(\mathrm{HCl})$ is required as an essential cocatalyst anyway, ${ }^{32,43}$ this hypothesis seemed unlikely. The protonation of the substrate with $\mathrm{HCl}$ in solution would deliver an ion pair. Therefore, we speculated that the inability of capsule II to encapsulate ion pairs (see discussion above) is the main reason for its inability to catalyze reactions. ${ }^{43}$ In the case of iminium catalysis, where the iminium ion is encapsulated as ion pair, this remains to be our best hypothesis to explain capsule II's inability to influence the enantioselectivity of the reaction. However, in the case of acid-catalyzed reactions, unpublished results with a series of derivatives of capsules I and II indicate an alternative explanation. After corroborating these findings, we expect to publish these findings in due course.

\section{Summary and Outlook}


Our curiosity-driven research into the puzzling encapsulation behaviors of the supramolecular capsules I and II triggered the discovery of a series of reactions that can be catalyzed inside I. The acetal hydrolysis performed inside the capsule displayed good size selectivity, which cannot be achieved in solution. Furthermore, we demonstrated that iminium catalysis can be performed inside capsule $\mathbf{I}$. The reactive iminium ion is encapsulated efficiently due to cation- $\pi$ interactions and has to react in the confined environment. This resulted in increased enantioselectivities (up to a $\Delta$ ee of 92\%). Most surprisingly, the tail-to-head terpene cyclization, which provides the vast variety of cyclic terpene natural products, was mimicked with the catalyst combination of capsule I and $\mathrm{HCl}$ in the case of monoterpenes. The natural products eucalyptol and $\alpha$-terpinene, which so far were not accessible in a one-pot procedure from acyclic terpene precursors were formed in $39 \%$ and 35\% yield, respectively. Terpene cyclizations continue to be one main focus of our research and the results of sesquiterpene cyclizations are about to be published. Further efforts will be devoted to developing less symmetric capsules to influence the conformation of the bound substrate. Additionally, we reported that the carbonyl-olefin metathesis can be achieved using the cocatalytic system of capsule $\mathbf{I}$ and $\mathrm{HCl}$. These results were surprising since $\mathrm{HCl}$ alone in solution was reported to be an ineffective catalyst for this reaction. Although a wide range of further reactions can be accelerated inside capsule $\mathbf{I}$, we currently focus on reactions where the capsule modulates reaction selectivity. Only in these cases, we expect capsule catalysis to have a potential synthetic application.

Our research, summarized in this article, as well as results from other groups demonstrated that certain supramolecular capsules are promising catalysts. They are able to confer different selectivities onto the reaction product as compared to regular solution experiments. Nevertheless, most studies so far constitute basic research with little synthetic relevance. To become more 
synthetically relevant, further research is certainly required. First, the prerequisites for catalytic activity have to be fully understood. Afterwards, the design of novel, more selective supramolecular hosts will be the focus, which potentially should lead to more selective, and, therefore, more synthetically relevant examples.

\section{AUTHOR INFORMATION}

\section{Corresponding Author}

Email: konrad.tiefenbacher@unibas.ch, tkonrad@ethz.ch

\section{ORCID}

Qi Zhang: 0000-0001-7435-1186

Lorenzo Catti: 0000-0003-0727-0620

Konrad Tiefenbacher: 0000-0002-3351-6121

\section{Present Addresses}

Lorenzo Catti: Laboratory for Chemistry and Life Science, Institute of Innovative Research, Tokyo Institute of Technology, 4259 Nagatsuta, Midori-ku, Yokohama 226-8503, Japan.

\section{ACKNOWLEDGMENT}

This work was supported by funding from the European Research Council Horizon 2020 Programme [ERC Starting Grant 714620-TERPENECAT], the Swiss National Science Foundation as part of the NCCR Molecular Systems Engineering and the Bayerische Akademie der Wissenschaften (Junges Kolleg). L. C. thanks JSPS and the Alexander von Humboldt Foundation for a postdoctoral fellowship. 


\section{Biographical Information}

Qi Zhang obtained his master degree in 2012 at the Technical University of Munich and began his Ph.D. studies under the supervision of Prof. Konrad Tiefenbacher in the same year. After receiving his Ph.D degree at the Technical University of Munich in 2016, he re-joined the research group of Prof. Konrad Tiefenbacher at the University of Basel as a postdoctoral fellow. In late 2018, he will start his independent career at Sichuan University in Chengdu, China.

Lorenzo Catti received his master degree in organic chemistry from the Technical University of Munich in 2013 under the supervision of Prof. Konrad Tiefenbacher. In 2016, he moved with the Tiefenbacher group to the University of Basel and, in 2017, completed his Ph.D. research on the application and development of resorcinarene-based supramolecular catalysts. He is currently a JSPS/AvH postdoctoral fellow in the Yoshizawa research group at the Tokyo Institute of Technology.

Konrad Tiefenbacher is a dual tenure track Assistant Professor of Chemistry at the University of Basel and the ETH Zürich. He obtained his master degree at the Technical University of Vienna in 2004 and the Ph.D. degree at the University of Vienna in the group of Prof. Johann Mulzer in 2009. He conducted postdoctoral research with Prof. Julius Rebek at The Scripps Research Institute in La Jolla in 2010/2011. In December 2011 he joined the faculty of TU Munich as assistant professor (no tenure track) before switching to his current position in 2016.

\section{REFERENCES}

1. Kirby, A. J., Enzyme mechanisms, models, and mimics. Angew. Chem. Int. Ed. 1996, 35, 707-724.

2. Motherwell, W. B.; Bingham, M. J.; Six, Y., Recent progress in the design and synthesis of artificial enzymes. Tetrahedron 2001, 57, 4663-4686.

3. Pauling, L., Nature of forces between large molecules of biological interest. Nature 1948, 161, 707-709.

4. Fried, S. D.; Boxer, S. G., Electric Fields and Enzyme Catalysis. Annu. Rev. Biochem 2017, 86, 387-415. 
5. Kamerlin, S. C. L.; Warshel, A., At the dawn of the 21st century: Is dynamics the missing link for understanding enzyme catalysis? Proteins: Structure, Function, and Bioinformatics 2010, 78, 1339-1375.

6. Catti, L.; Zhang, Q.; Tiefenbacher, K., Self-Assembled Supramolecular Structures as Catalysts for Reactions Involving Cationic Transition States. Synthesis 2016, 48, 313-328.

7. Pluth, M. D.; Bergman, R. G.; Raymond, K. N., Proton-Mediated Chemistry and Catalysis in a Self-Assembled Supramolecular Host. Acc. Chem. Res. 2009, 42, 1650-1659.

8. Leenders, S. H. A. M.; Gramage-Doria, R.; de Bruin, B.; Reek, J. N. H., Transition metal catalysis in confined spaces. Chem. Soc. Rev. 2015, 44, 433-448.

9. Catti, L.; Zhang, Q.; Tiefenbacher, K., Advantages of Catalysis in Self-Assembled Molecular Capsules. Chem. Eur. J. 2016, 22, 9060-9066.

10. Zarra, S.; Wood, D. M.; Roberts, D. A.; Nitschke, J. R., Molecular containers in complex chemical systems. Chem. Soc. Rev. 2015, 44, 419-432.

11. Yoshizawa, M.; Klosterman, J. K.; Fujita, M., Functional Molecular Flasks: New Properties and Reactions within Discrete, Self-Assembled Hosts. Angew. Chem. Int. Ed. 2009, 48, 3418-3438.

12. Wiester, M. J.; Ulmann, P. A.; Mirkin, C. A., Enzyme Mimics Based Upon Supramolecular Coordination Chemistry. Angew. Chem. Int. Ed. 2011, 50, 114-137.

13. Vriezema, D. M.; Comellas Aragonès, M.; Elemans, J. A. A. W.; Cornelissen, J. J. L. M.; Rowan, A. E.; Nolte, R. J. M., Self-Assembled Nanoreactors. Chem. Rev. 2005, 105, 1445-1490. 
14. Raynal, M.; Ballester, P.; Vidal-Ferran, A.; van Leeuwen, P. W. N. M., Supramolecular catalysis. Part 2: artificial enzyme mimics. Chem. Soc. Rev. 2014, 43, 1734-1787.

15. Otte, M., Size-Selective Molecular Flasks. ACS Catal. 2016, 6, 6491-6510.

16. Meeuwissen, J.; Reek, J. N. H., Supramolecular catalysis beyond enzyme mimics. Nat. Chem. 2010, 2, 615-621.

17. Marchetti, L.; Levine, M., Biomimetic Catalysis. ACS Catal. 2011, 1, 1090-1118.

18. Koblenz, T. S.; Wassenaar, J.; Reek, J. N. H., Reactivity within a confined self-assembled nanospace. Chem. Soc. Rev. 2008, 37, 247-262.

19. Brown, C. J.; Toste, F. D.; Bergman, R. G.; Raymond, K. N., Supramolecular Catalysis in Metal-Ligand Cluster Hosts. Chem. Rev. 2015, 115, 3012-3035.

20. MacGillivray, L. R.; Atwood, J. L., A chiral spherical molecular assembly held together by 60 hydrogen bonds. Nature 1997, 389, 469-472.

21. Avram, L.; Cohen, Y., Spontaneous Formation of Hexameric Resorcinarene Capsule in Chloroform Solution as Detected by Diffusion NMR. J. Am. Chem. Soc. 2002, 124, 15148-15149.

22. Avram, L.; Cohen, Y.; Rebek, J., Recent advances in hydrogen-bonded hexameric encapsulation complexes. Chem. Commun. 2011, 47, 5368-5375.

23. Yamanaka, M.; Shivanyuk, A.; Rebek, J., Kinetics and Thermodynamics of Hexameric Capsule Formation. J. Am. Chem. Soc. 2004, 126, 2939-2943. 
24. Shivanyuk, A.; Rebek, J., Reversible encapsulation by self-assembling resorcinarene subunits. Proc. Natl. Acad. Sci. U.S.A. 2001, 98, 7662-7665.

25. Avram, L.; Cohen, Y., Discrimination of Guests Encapsulation in Large Hexameric Molecular Capsules in Solution: Pyrogallol[4]arene versus Resorcin[4]arene Capsules. J. Am. Chem. Soc. 2003, 125, 16180-16181.

26. Avram, L.; Cohen, Y., Self-Recognition, Structure, Stability, and Guest Affinity of Pyrogallol[4]arene and Resorcin[4]arene Capsules in Solution. J. Am. Chem. Soc. 2004, 126, 11556-11563.

27. Gerkensmeier, T.; Iwanek, W.; Agena, C.; Frohlich, R.; Kotila, S.; Nather, C.; Mattay, J., Self-assembly of 2,8,14,20-tetraisobutyl-5,11,17,23-tetrahydroxyresorc[4]arene. Eur. J. Org. Chem. 1999, 2257-2262.

28. Cavarzan, A.; Scarso, A.; Sgarbossa, P.; Strukul, G.; Reek, J. N. H., Supramolecular Control on Chemo- and Regioselectivity via Encapsulation of (NHC)-Au Catalyst within a Hexameric Self-Assembled Host. J. Am. Chem. Soc. 2011, 133, 2848-2851.

29. Zhang, Q.; Tiefenbacher, K., Hexameric Resorcinarene Capsule is a Brønsted Acid: Investigation and Application to Synthesis and Catalysis. J. Am. Chem. Soc. 2013, 135, 1621316219.

30. Pellegrino, L. M.; Carmen, T.; Giuseppe, F.; Margherita, D. R.; Annunziata, S.; Antonio, R.; Carmine, G.; Placido, N., Mild Friedel-Crafts Reactions inside a Hexameric Resorcinarene Capsule: $\mathrm{C}-\mathrm{Cl}$ Bond Activation through Hydrogen Bonding to Bridging Water Molecules. Angew. Chem. 2018, 130, 5521-5526. 
31. Zhang, Q.; Catti, L.; Kaila, V. R. I.; Tiefenbacher, K., To catalyze or not to catalyze: elucidation of the subtle differences between the hexameric capsules of pyrogallolarene and resorcinarene. Chem. Sci. 2017, 8, 1653-1657.

32. M., K. J.; Konrad, T., Elucidating the Importance of Hydrochloric Acid as a Cocatalyst for Resorcinarene - Capsule - Catalyzed Reactions. ChemCatChem, 10.1002/cctc.201800326.

33. Tunstad, L. M.; Tucker, J. A.; Dalcanale, E.; Weiser, J.; Bryant, J. A.; Sherman, J. C.; Helgeson, R. C.; Knobler, C. B.; Cram, D. J., Host-guest complexation. 48. Octol building blocks for cavitands and carcerands. J. Org. Chem. 1989, 54, 1305-1312.

34. Erkkilä, A.; Majander, I.; Pihko, P. M., Iminium Catalysis. Chem. Rev. 2007, 107, 54165470.

35. Bräuer, T. M.; Zhang, Q.; Tiefenbacher, K., Iminium Catalysis inside a Self-Assembled Supramolecular Capsule: Modulation of Enantiomeric Excess. Angew. Chem. Int. Ed. 2016, 55, 7698-7701.

36. Bräuer, T. M.; Zhang, Q.; Tiefenbacher, K., Iminium Catalysis inside a Self-Assembled Supramolecular Capsule: Scope and Mechanistic Studies. J. Am. Chem. Soc. 2017, 139, 1750017507.

37. Pollok, C. H.; Zhang, Q.; Tiefenbacher, K.; Merten, C., Chirality Induction from a Chiral Guest to the Hydrogen-Bonding Network of Its Hexameric Resorcinarene Host Capsule. ChemPhysChem 2017, 18, 1987-1991. 
38. Pronin, S. V.; Shenvi, R. A., Synthesis of highly strained terpenes by non-stop tail-to-head polycyclization. Nat. Chem. 2012, 4, 915-920.

39. Yoder, R. A.; Johnston, J. N., A Case Study in Biomimetic Total Synthesis: Polyolefin Carbocyclizations to Terpenes and Steroids. Chem. Rev. 2005, 105, 4730-4756.

40. Lesburg, C. A.; Zhai, G.; Cane, D. E.; Christianson, D. W., Crystal Structure of Pentalenene Synthase: Mechanistic Insights on Terpenoid Cyclization Reactions in Biology. Science 1997, 277, 1820-1824.

41. Starks, C. M.; Back, K.; Chappell, J.; Noel, J. P., Structural Basis for Cyclic Terpene Biosynthesis by Tobacco 5-Epi-Aristolochene Synthase. Science 1997, 277, 1815-1820.

42. Zhang, Q.; Tiefenbacher, K., Terpene cyclization catalysed inside a self-assembled cavity. Nat. Chem. 2015, 7, 197-202.

43. Zhang, Q.; Catti, L.; Pleiss, J.; Tiefenbacher, K., Terpene Cyclizations inside a Supramolecular Catalyst: Leaving-Group-Controlled Product Selectivity and Mechanistic Studies. J. Am. Chem. Soc. 2017, 139, 11482-11492.

44. Catti, L.; Tiefenbacher, K., Intramolecular hydroalkoxylation catalyzed inside a selfassembled cavity of an enzyme-like host structure. Chem. Commun. 2015, 51, 892-894.

45. Catti, L.; Pöthig, A.; Tiefenbacher, K., Host-Catalyzed Cyclodehydration-Rearrangement Cascade Reaction of Unsaturated Tertiary Alcohols. Adv. Synth. Catal. 2017, 359, 1331-1338. 
46. Catti, L.; Tiefenbacher, K., Brønsted Acid - Catalyzed Carbonyl - Olefin Metathesis inside a Self - Assembled Supramolecular Host. Angew. Chem. Int. Ed., doi:10.1002/anie.201712141.

47. Ludwig, J. R.; Zimmerman, P. M.; Gianino, J. B.; Schindler, C. S., Iron(III)-catalysed carbonyl-olefin metathesis. Nature 2016, 533, 374.

48. Ludwig, J. R.; Phan, S.; McAtee, C. C.; Zimmerman, P. M.; Devery, J. J.; Schindler, C. S., Mechanistic Investigations of the Iron(III)-Catalyzed Carbonyl-Olefin Metathesis Reaction. J. Am. Chem. Soc. 2017, 139, 10832-10842.

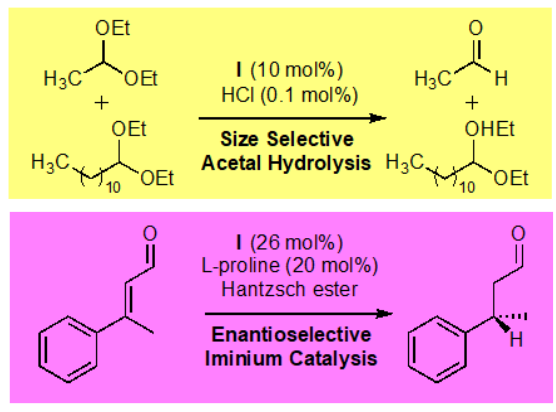

Infuencing Selectivity via Encapsulation

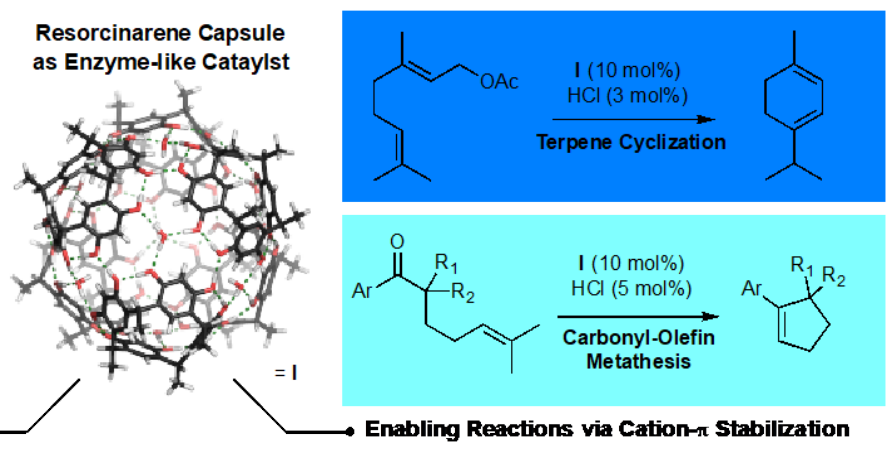

